\title{
ON MAPPINGS WHICH COMMUTE WITH CONVOLUTION
}

\author{
S. R. HARASYMIV \\ (Received 9 June, revised 28 October 1969) \\ Communicated by J. B. Miller
}

\section{Notation}

The symbol $\boldsymbol{D}$ will be written for the space of indefinitely differentiable functions on the $n$-dimensional Euclidean space $R^{n}$ which have compact support and $D^{\prime}$ will denote the space of Schwartz distributions on $R^{n}$, the topological dual of $D$. Except where the contrary is explicitly stated, it will be assumed that $D^{\prime}$ is equipped with the strong topology $\beta\left(\boldsymbol{D}^{\prime}, \boldsymbol{D}\right)$ induced by $\boldsymbol{D}$.

\section{Definitions}

We shall always use the term space of distributions (or, more briefly, distribution space) to mean a vector subspace of $D^{\prime}$ which contains the subspace $\boldsymbol{D}$. This convention will help us to avoid much tedious repetition.

2.1. Definition. Suppose that $E$ and $E^{\prime}$ are spaces of distributions and that $\langle$,$\rangle is a bilinear form on E \times E^{\prime}$ such that the relations

$$
\begin{aligned}
& \langle u, \varphi\rangle=u * \varphi(0) \quad(u \in E) \\
& \langle\varphi, v\rangle=\varphi * v(0) \quad\left(v \in E^{\prime}\right)
\end{aligned}
$$

hold whenever $\varphi$ is an element of $\boldsymbol{D}$. Then the ordered pair $\left(E, E^{\prime}\right)$ together with the bilinear form $\langle$,$\rangle is called a dual pair of distribution spaces. We shall usually$ omit explicit reference to the bilinear form $\langle$,$\rangle and speak simply of the dual$ pair $\left(E, E^{\prime}\right)$.

The next definition was introduced by Yoshinaga and Ogata in [1]. We restate it here for the sake of completeness.

2.2. Definition. (Yoshinaga and Ogata [1]). Suppose that $E$ is a locally convex space of distributions which possesses the following two properties:

(i) $D$ is dense in $E$.

(ii) The injection mappings $D \rightarrow E$ and $E \rightarrow D^{\prime}$ are continuous.

Then $E$ is said to be an admissible space. 
Remark. The topological dual $E^{\prime}$ of an admissible space $E$ will always be identified with a space of distributions in such as way that $\left(E, E^{\prime}\right)$ (with the bilinear form arising from the natural pairing of $E$ and $\left.E^{\prime}\right)$ ) is a dual pair of distribution spaces. This (unique) embedding of $E^{\prime}$ in $D^{\prime}$ will be called the natural embedding.

We now introduce the spaces with which we shall be concerned in this note.

2.3. Definition. Let $E$ be an admissible space. We say that $E$ is of type (c) (or a (c)-space) if it is a module over $\boldsymbol{D}$ (with respect to convolution) and has the following properties:

(i) For each $u \in E$, the mapping $\varphi \rightarrow u * \varphi(\varphi \in D)$ of $\boldsymbol{D}$ into $E$ is continuous.

(ii) For each $\varphi \in D$, the mapping $u \rightarrow u * \varphi(u \in E)$ of $E$ into itself is continuous.

2.4. Definition. Let $E$ be a $(c)$-space. A linear mapping $T$ of $E$ into $D^{\prime}$ is said to commute with convolution if

$$
T(u * \varphi)=(T u) * \varphi \quad(\varphi \in D, u \in E)
$$

The space of all such continuous mappings is denoted by $H_{c}\left(E, D^{\prime}\right)$.

Remark. Let $E$ be a $(c)$-space. If $u \in E$ and $T \in H_{c}\left(E, D^{\prime}\right)$, then we shall denote by $u * T$ the image of $u$ under the mapping $T$. With this notation, the convolution commutativity of $T$ is expressed by

$$
(u * \varphi) \bar{*} T=(u * T) * \varphi \quad(\varphi \in D, u \in E)
$$

$H_{c}\left(E, D^{\prime}\right)$ will always be identified with a space of distributions in such a way that the relations

$$
\begin{array}{ll}
u * \varphi=u * \varphi & (u \in E) \\
\varphi \bar{*}=\varphi * w & \left(w \in H_{c}\left(E, D^{\prime}\right)\right)
\end{array}
$$

hold whenever $\varphi$ is an element of $D$. This (unique) embedding of $H_{c}\left(E, D^{\prime}\right)$ in $D^{\prime}$ will be called the natural embedding.

\section{Some preliminary results}

3.1. Proposition. Suppose that $\left(E, E^{\prime}\right)$ is a dual pair of distribution spaces and that $T$ is a continuous linear mapping of $\mathbf{D}^{\prime}$ into itself which commutes with convolution (by elements of $\mathrm{D}$ ). Then the following assertions are equiavlent to one another:

(1) $E$ is invariant under $T$ and the mapping $u \rightarrow T u(u \in E)$ of $E$ into itself is weakly continuous.

(2) Both $E$ and $E^{\prime}$ are invariant under $T$ and

$$
\langle T u, v\rangle=\langle u, T v\rangle \quad\left(u \in E, v \in E^{\prime}\right)
$$


Proof. If we bear in mind the theorem in Section 5.11.3 of Edwards [2], it is simple to show that the mapping $v \rightarrow T v\left(v \in E^{\prime}\right)$ is the adjoint of the mapping $u \rightarrow T u(u \in E)$ of $E$ into itself.

3.2. Proposition. Suppose that $E$ is a (c)-space and that $T$ is a continuous linear mapping of $D^{\prime}$ into itself which commutes with convolution (by elements of $D)$. Then $H_{c}\left(E, D^{\prime}\right)$ is invariant under $T$ and

$$
u \bar{*} T w=T(u \approx w) \quad\left(u \in E, w \in H_{c}\left(E, D^{\prime}\right)\right)
$$

Proof. Once again using the theorem in Section 5.11.3 of Edwards [2], it is not difficult to demonstrate that the mapping $u \rightarrow T(u * w)(u \in E)$ defines an element of $H_{c}\left(E, D^{\prime}\right)$. The latter is readily shown to be just $T w$.

As an adjunct to Proposition 3.2, we have the following result. Its proof is along the same lines as that of Proposition 3.2.

3.3. Proposition. Let $T$ be a continuous linear mapping of $D^{\prime}$ into itself which commutes with convolution (by elements of $D$ ). Suppose that $E$ is a (c)-space with the following property:

(i) $E$ is invariant under $T$ and the mapping $u \rightarrow T u(u \in E)$ of $E$ into itself is continuous.

It then follows that

$$
(T u) * w=T(u \bar{*} w)=u \bar{*}(T w) \quad\left(u \in E, w \in H_{c}\left(E, D^{\prime}\right)\right)
$$

The foregoing three propositions easily yield the following facts about spaces of type $(c)$. These will be needed later.

3.4. Proposition. Suppose that $E$ is a (c)-space. Then the following assertions are true:

(1) $E^{\prime}$ is a module over $D$ and for each $\varphi \in D$

$$
\langle u * \varphi, v\rangle=\langle u, v * \varphi\rangle \quad\left(u \in E, v \in E^{\prime}\right)
$$

(2) $H_{c}\left(E, D^{\prime}\right)$ is a module over $D$ and for each $\varphi \in D$

$$
(u * \varphi) * w=(u * w) * \varphi=u *(w * \varphi) \quad\left(u \in E, w \in H_{c}\left(E, D^{\prime}\right)\right)
$$

\section{A criterion for mappings which commute with convolution}

In this section we shall take a look at a condition which determines whether a distribution $w \in D^{\prime}$ is an element of $H_{c}\left(E, D^{\prime}\right)$. We need the next result to do this.

4.1. Proposition. Suppose that $E$ is a (c)-space and that $w \in H_{c}\left(E, D^{\prime}\right)$. Then the following assertion is true:

(1) $w * \varphi \in E^{\prime}$ for each $\varphi \in D$ and the mapping $\varphi \rightarrow w * \varphi(\varphi \in D)$ of $\boldsymbol{D}$ into $E^{\prime}$ is weakly continuous. 
Moreover, we have the identity

$$
\langle u, w * \varphi\rangle=(u * w) * \varphi(0) \quad(\varphi \in D, u \in E)
$$

ProOF. The mapping $\varphi \rightarrow w * \varphi(\varphi \in D)$ is just the adjoint of the continuous linear mapping $u \rightarrow u{ }^{*} w(u \in E)$ of $E$ into $D^{\prime}$.

If the space $E$ has the topology $\tau\left(E, E^{\prime}\right)$, then the preceding result has a converse. We have then the following criterion for determining whether a distribution $w$ belongs to $H_{c}\left(E, D^{\prime}\right)$.

4.2. Theorem. Suppose that $E$ is a (c)-space which has the topology $\tau\left(E, E^{\prime}\right)$ and that $w \in D^{\prime}$. Then the following two conditions are equivalent:

(1) $w \in H_{c}\left(E, D^{\prime}\right)$

(2) $w * \varphi \in E^{\prime}$ for all $\varphi \in D$ and the mapping $\varphi \rightarrow w * \varphi(\varphi \in D)$ of $D$ into $E^{\prime}$ is weakly continuous.

ProOF. We need only show that (2) implies (1). Consider the adjoint of the mapping $\varphi \rightarrow w * \varphi(\varphi \in D)$, which is a linear mapping of $E$ into $D^{\prime}$. This adjoint is continuous (because $E$ has the topology $\tau\left(E, E^{\prime}\right)$ ) and commutes with convolution (because of Proposition 3.4). It is therefore represented by a distribution in $H_{c}\left(E, D^{\prime}\right)$ and this distribution is easily shown to be precisely $w$.

4.3. Corollary. Suppose that $E$ is a (c)-space which has the topology $\tau\left(E, E^{\prime}\right)$. Then $E^{\prime}$ is contained in $H_{c}\left(E, D^{\prime}\right)$.

An additional restriction on $E$, which in itself is not too severe, enables us to strengthen considerably the content of Theorem 4.2. We now turn our attention to this task, beginning with a couple of lemmas.

4.4. Lemma. Suppose that $E$ is a (c)-space and that $u \in E$. Let $w \in D^{\prime}$ be $a$ distribution which has the following property:

(i) $w * \varphi \in H_{c}\left(E, D^{\prime}\right)$ for each $\varphi \in D$.

Then there exists a distribution $s \in D^{\prime}$ such that

$$
u *(w * \varphi)=s * \varphi \quad(\varphi \in D) .
$$

Proof. Let $u$ and $w$ be as in the statement of the Lemma. Denote by $L$ the mapping of $\boldsymbol{D}$ into $\boldsymbol{D}^{\prime}$ which is defined by

$$
L \varphi=u \bar{*}(w * \varphi) \quad(\varphi \in \boldsymbol{D}) .
$$

We claim that $L$ is continuous from $D$ into $D^{\prime}$. To verify this, notice that, since $D$ has its Mackey topology $\tau\left(D, D^{\prime}\right)$, the continuity of the mapping $L$ will be established if we demonstrate that it is weakly continuous. Now, in view of the hypothesis about the distribution $w \in D^{\prime}$, Proposition 4.1 tells us that $w * \varphi * \psi \in E^{\prime}$ for all $\varphi, \psi \in D$; and that

$$
\langle u, w * \varphi * \psi\rangle=(u *(w * \varphi)) * \psi(0) \quad(\varphi, \psi \in D) .
$$


Relations (4.7) and (4.8) entail that for each $\psi \in D$

$$
L \varphi * \psi(0)=(u *(w * \psi)) * \varphi(0) \quad(\varphi \in D)
$$

and the weak continuity of $L$ (as a mapping of $D$ into $D^{\prime}$ ) is now evident.

Next, we notice that $L$ commutes with convolution; this is an immediate consequence of Proposition 3.4 (2).

Having established the continuity and convolution commutativity of $L$, we infer the existence of a distribution $s \in D^{\prime}$ such that

$$
L \varphi=s * \varphi \quad(\varphi \in D) .
$$

In view of (4.7), we see that (4.10) expresses the desired result.

4.5. Lemma. Suppose that $E$ is a $(c)$-space which has the topology $\tau\left(E, E^{\prime}\right)$ and whose dual $E^{\prime}$ is sequentially complete for the topology $\beta\left(E^{\prime}, E\right)$. Let $w \in D^{\prime}$ be a distribution which has the following property:

(i) $w * \varphi \in H_{c}\left(E, D^{\prime}\right)$ for each $\varphi \in D$. .

Then $w$ is an element of $H_{c}\left(E, D^{\prime}\right)$.

Proof. Let $w$ be a distribution which has property (i) above. Choose a countable approximate identity $\left(k_{m}\right)$ in $\boldsymbol{D}^{\prime}$ consisting of functions in $\boldsymbol{D}$. By Proposition 4.1, $w * \varphi * \psi \in E^{\prime}$ for all $\varphi, \psi \in D$. Therefore, for each positive integer $m$, we may define a mapping $L_{m}$ of $D$ into $E^{\prime}$ by setting

$$
L_{m} \varphi=w * \varphi * k_{m} \quad(\varphi \in D) .
$$

Our first claim is that, for each $m$, the mapping $L_{m}$ is strongly continuous from $D$ into $E^{\prime}$. This is easy to verify. For, in view of the hypothesis about $w$, reference to Proposition 4.1 assures us that, for each $m, L_{m}$ is weakly, and hence also strongly, continuous. (The assertion about the strong continuity of each $L_{m}$ is justified by Proposition 8.6.5 in Edwards [2]). We notice also that relations (4.11) and (4.1) entail that for each $m$ and each $u \in E$

$$
\left\langle u, L_{m} \varphi\right\rangle=\left(u \bar{*}\left(w * k_{m}\right)\right) * \varphi(0) \quad(\varphi \in D) .
$$

We next remark that $\left(L_{m}\right)$, as a sequence of mappings of $D$ into $E^{\prime}$, is bounded at each point of $\boldsymbol{D}$ when $E^{\prime}$ has the topology $\beta\left(E^{\prime}, E\right)$. To show this, it is sufficient to demonstrate that, for each $\varphi \in D,\left(L_{m} \varphi\right)$ is uniformly bounded on each bounded subset of $E$. Thus consider an arbitrary (but fixed) element $\varphi \in D$; and let $B$ be a bounded subset of $E$. For each $u \in E$, let $s_{u}$ be the distribution in $D^{\prime}$ which satisfies

$$
u \bar{*}(w * \psi)=s_{u} * \psi \quad(\psi \in D)
$$

The existence of such distributions $s_{u}$ is guaranteed by Lemma 4.4. Observe that, for each $\psi \in D$, the set $\left\{s_{u} * \psi: u \in B\right\}$ is the image in $D^{\prime}$ of the set $B$ under the continuous mapping $u \rightarrow u *(w * \psi)(u \in E)$ of $E$ into $D^{\prime}$. Since $B$ is bounded in $E$ 
we conclude that $\left\{s_{u} * \psi: u \in B\right\}$ is bounded in $D^{\prime}$ for each $\psi \in D$. Théorème XXII in Chapitre VI of Schwartz [3] now ensures that the set $\left\{s_{u}: u \in B\right\}$ is bounded in $\boldsymbol{D}^{\prime}$. Thus, since the sequence $\left(\varphi * k_{m}\right)$ is convergent in $\boldsymbol{D}$ and therefore uniformly bounded on each bounded subset of $D^{\prime}$, we conclude that there exists a constant $M$ such that

$$
\left|\left\langle s_{u} * \varphi * k_{m}(0)\right\rangle\right| \leqq M \quad(k=1,2, \cdots)
$$

uniformly for $u \in B$. In view of (4.12), (4.13) and (4.14), we may now assert that

$$
\left|\left\langle u, L_{m} \varphi\right\rangle\right| \leqq M \quad(k=1,2, \cdots)
$$

uniformly for $u \in B$. The pointwise boundedness of the sequence $\left(L_{m}\right)$ of mappings of $D$ into $E^{\prime}$ has now been established.

Now let $H_{0}$ be the subspace of $D$ which consists of all elements $\varphi \in D$ for which $\left(L_{m} \varphi\right)$ converges strongly in $E^{\prime}$. We shall show that $H_{0}$ coincides with the whole of $D$. Since $E^{\prime}$ is strongly sequentially complete and $D$ is barrelled for its strong topo$\operatorname{logy} \beta\left(D, D^{\prime}\right)$, we need only show that $H_{0}$ is dense in $\boldsymbol{D}$ (Edwards [2], Corollary 7.1.4). This, in turn, will be established if we succeed in demonstrating that $\varphi * \psi \in H_{0}$ whenever $\varphi \in D$ and $\psi \in D$. To verify that this in fact true, we proceed as follows. First we notice that if $\psi \in D$, then

$$
\lim _{m} \psi * k_{m}=\psi \text { strongly in } D .
$$

Now, Proposition 4.1 tells us that if $\varphi, \psi \in D$, then $w * \varphi * \psi \in E^{\prime}$; and that for each fixed $\varphi \in \boldsymbol{D}$, the maping $\psi \rightarrow w * \varphi * \psi(\psi \in \boldsymbol{D})$ is weakly, and hence strongly, continuous from $D$ into $E^{\prime}$. In view of (4.16) we may therefore conclude that if $\varphi, \psi \in D$, then

$$
\lim _{m} L_{m}(\varphi * \psi)=\lim _{m} w * \varphi * \psi * k_{m}=w * \varphi * \psi
$$

the limits in (4.17) being in the strong topology $\beta\left(E^{\prime}, E\right)$ on $E^{\prime}$. As was explained above, we may now assert that $H_{0}=D$.

We can now define a mapping $L$ of $D$ into $E^{\prime}$ by the relation

$$
L \varphi=\lim _{m} L_{m} \varphi \quad(\varphi \in D)
$$

the limit in (4.18) being once again a strong limit in $E^{\prime}$. Then $L$ is strongly continuous from $D$ into $E^{\prime}$ (we have again used Corollary 7.1.4 in Edwards [2]).

We are now in a position to complete our proof. According to Theorem 4.2, it is sufficient to show that the mapping $\varphi \rightarrow w * \varphi(\varphi \in \boldsymbol{D})$ is a weakly continuous linear mapping of $\boldsymbol{D}$ into $E^{\prime}$; it will then follow that $w$ is indeed an element of $H_{c}\left(E, D^{\prime}\right)$. Now, since $D^{\prime}$ is reflexive, each strongly continuous linear mapping of $D$ into $E^{\prime}$ is weakly continuous (Edwards [2], Corollary 8.6.7). Therefore, we need establish only that the mapping $\varphi \rightarrow w * \varphi(\varphi \in D)$ is strongly continuous from $\boldsymbol{D}$ into $E^{\prime}$. But if $\varphi \in \boldsymbol{D}$ and $\psi \in \boldsymbol{D}$, th en we see that 


$$
\begin{aligned}
\psi * L \varphi(0) & =\langle\psi, L \varphi\rangle \\
& =\lim _{m}\left(\psi, L_{m} \varphi\right\rangle \\
& =\lim _{m}\left\langle\psi, w * \varphi * k_{m}\right\rangle \\
& =\lim _{m} \psi * w * \varphi * k_{m}(0) \\
& =\psi * w * \varphi(0) .
\end{aligned}
$$

We infer that $L \varphi=w * \varphi$ for each $\varphi \in D$; whence it follows immediately that the mapping $\varphi \rightarrow w * \varphi(\varphi \in \boldsymbol{D})$ is a strongly continuous mapping of $\boldsymbol{D}$ into $E^{\prime}$.

With the aid of the above two lemmas, we can prove the following variant of Theorem 4.2.

4.6. THEOREM. Suppose that $E$ is a $(c)$-space which has the topology $\tau\left(E, E^{\prime}\right)$ and whose dual $E^{\prime}$ is sequentially complete for the topology $\beta\left(E^{\prime}, E\right)$. Let $w \in D^{\prime}$ be a distribution. Then the following three conditions are equivalent to one another:

(1) $w \in H_{c}\left(E, D^{\prime}\right)$.

(2) $w * \varphi \in E^{\prime}$ for each $\varphi \in D$.

(3) $w * \varphi * \psi \in E^{\prime}$ for all $\varphi, \psi \in D$.

Proof. Theorem 4.2 ensures that (2) holds if $w \in D^{\prime}$ satisfies (1). Proposition 3.4 entails that (2) implies (3). To complete the proof, notice first that $E^{\prime}$ is contained in $H_{c}\left(E, D^{\prime}\right)$ (Corollary 4.3 ). Thus if (3) holds, we may appeal to Lemma 4.5 and deduce that $w * \varphi \in H_{c}\left(E, D^{\prime}\right)$ for each $\varphi \in D$; whence it follows (again by Lemma 4.5) that (1) holds.

REMARK. Proposition 4.6 is applicable to $(c)$-spaces $E$ which are either barrelled or bornological; see Section 8.4.13 in Edwards [2].

\section{References}

[1] K. Yoshinaga and H. Ogata, 'On convolutions', J. Sci. Hiroshima Univ., Ser. A, 22 (1958), 15-24.

[2] R. E. Edwards, Functional analysis: Theory and applications (Holt, Rinehart and Winston, New York, 1965).

[3] L. Schwartz, Théorie des distributions (Hermann, Paris, 1967).

Department of Mathematics

University of Connecticut 